\title{
A framework for the construction of level set methods for shape optimization and reconstruction
}

\author{
MARTIN BURGER ${ }^{\dagger}$ \\ Industrial Mathematics Institute, Johannes Kepler Universität Linz \\ Altenbergerstr. 69, 4040 Linz, Austria
}

[Received 24 September 2002 and in revised form 10 March 2003]

\begin{abstract}
The aim of this paper is to develop a functional-analytic framework for the construction of level set methods, when applied to shape optimization and shape reconstruction problems. As a main tool we use a notion of gradient flows for geometric configurations such as used in the modelling of geometric motions in materials science. The analogies to this field lead to a scale of level set evolutions, characterized by the norm used for the choice of the velocity. This scale of methods also includes the standard approach used in previous work on this subject as a special case.

Moreover, we apply this framework to some (inverse) model problems for elliptic boundary value problems. In numerical experiments we demonstrate that an appropriate choice of norms (dependent on the problem) yields stable and fast methods.
\end{abstract}

Keywords: Level sets; shape optimization; shape reconstruction; inverse problems; Hamilton-Jacobi equations; materials science; crystal growth; gradient flows.

2000 Mathematics Subject Classification: 65J20, 49Q10, 49L99, 35C44, 35R30.

\section{Introduction}

Level set methods, originally introduced by Osher and Sethian [58], have developed to be one of the most successful tools for the computation of evolving geometries, which appear in many practical applications (we refer to the monographs by Osher and Fedkiw [56], Sethian [69], and the references therein for a presentation of various applications). Level set methods do not only lead to efficient computational schemes, but are also able to handle topological changes such as merging and splitting of connected components, which is impossible with classical methods based on curve parameterizations.

Since evolving geometries arise in many physical processes such as growth or phase transitions, materials science has been a major field of applications for level set methods from the beginning. Recently, level set methods have been employed also for the solution of shape optimization problems and shape reconstruction problems, i.e., inverse problems, where the unknown is some shape or curve. Whereas the choice of the normal speed of an evolving curve or surface is determined by a physical model in materials science, the situation is different for shape optimization and reconstruction, where one of the basic questions for the setup of a level set method is an appropriate choice of the velocity such that a decrease in some functional (and possibly convergence to a solution of the optimization or reconstruction problem) can be achieved. In most of the existing literature (cf. [7, 18, 22, 27, 28, 43, 50, 57, 63, 64, 66, 70]), the choice of the velocity is motivated by ad-hoc approaches, and the presentation is rather problem-specific or restricted to a small class of

\footnotetext{
${ }^{\dagger}$ Email: burger@indmath.uni-linz.ac.at
} 
problems. The aim of this paper is to make a first step on the way towards a unified theory of level set methods for inverse problems, which also involves a clear functional-analytic background for the construction of such methods. The main motivation for this step is to carry over techniques used for variational models in materials science, where the speed is defined as a gradient flow for a corresponding energy functional, to the field of shape optimization and shape reconstruction, thus providing a framework for the construction of level set methods for these problem classes.

Since most attention in this paper is paid to a framework for constructing level set methods for inverse problems, we shall not be concerned with other important issues in the context of level set methods, which we only mention briefly in the following. A first important issue for ill-posed problems is their regularization, which is usually realized by adding additional stabilizing terms to the objective (such as perimeter) or by an appropriate termination of the iteration dependent on the data noise. Another important problem is the coupling of level set methods and elliptic partial differential equations arising in most of the problems presented here, from an analytical as well as from a computational point of view. Both in materials science and in inverse problems, such a coupling usually occurs in two directions: the level set evolution is influenced by the solution of an underlying state equation via a functional dependence in the normal speed and vice versa influences the state equation via the zero level set on which the equation has to be solved, respectively on whose boundary some Dirichlet or Neumann conditions are posed.

For convenience, we will restrict our attention to the case $\Omega \subset \mathbb{R}^{2}$ in the following, since for $\Omega \subset \mathbb{R}^{1}$ the problems of shape optimization and reconstruction reduce to finite-dimensional problems and for $\Omega \subset \mathbb{R}^{d}, d \geqslant 3$, the technicalities in the differential geometry of surfaces might shadow some of the key features. Nonetheless, the main ideas presented here remain unchanged in arbitrary spatial dimensions.

The remainder of the paper is organized as follows: we first give a short introduction to level set methods and shape derivatives, which are the main tools used in the following. Motivated by the applications we have in mind (and the ones considered in previous work), we present three model problems related to underlying state equations of elliptic type in Section2, each of them representing a larger class of problems. We will outline the specific features of the different model problems as well as some common properties for all problem classes such as a unified representation of the shape derivative. In Section 3, a variational framework for the construction of local level set methods based on shape derivatives is derived, which is motivated by variational models in materials science. The application of this framework to our model problems and some properties of the arising level set methods are discussed in Section 4 Finally, we present the results of some numerical experiments for our model problems, before we conclude and give an outlook to important problems for future work in Section 5

\subsection{Basic notations}

In the following we introduce the basic notations and assumptions used throughout the paper. We shall use the following standard notations from geometric measure theory: $\mathcal{L}^{d}$ denotes the $d$ dimensional Lebesgue measure and $\mathcal{H}^{d}$ denotes the $d$-dimensional Hausdorff measure (cf. [31, 53] for detailed definitions and further properties of these measures). By $n$ we shall always denote the normal vector of a curve in $\mathbb{R}^{2}$, and by $\kappa=\operatorname{div} n$ its curvature. The indicator function of a set $A$ will be denoted by $\chi_{A}$, where

$$
\chi_{A}= \begin{cases}1 & \text { in } A, \\ 0 & \text { else } .\end{cases}
$$


For open or closed sets $K \subset \mathbb{R}^{d}$ we will use the standard notions of continuity and differentiability, denoting the total derivative of order $j$ of a function $f$ by $D^{(j)} f$, and partial derivatives with respect to a variable $x$ by the standard symbol $\partial f / \partial x$, if $x \in \mathbb{R}^{N}$ is the spatial variable also by $\nabla f$. Moreover we shall use Sobolev spaces on domains $\Omega$ and on curves $\Gamma$, denoted as usual by $H^{r}(\Omega)$ or $H^{r}(\Gamma)$. For details on their definition and properties we refer to the monograph by Adams [2].

\subsection{Level set methods}

The key feature of the level set approach is to represent domains and their boundaries not via parameterizations, but as level sets of a continuous function $\phi$, the so-called level set function.

For the computation of an evolving open set $\Omega(t), t \in \mathbb{R}^{+}$, one can define the function $\phi$ on $\mathbb{R}^{N} \times \mathbb{R}_{+}$and determine the evolution of $\Omega$ via

$$
\Omega(t)=\{\phi(\cdot, t)<0\} .
$$

The boundary $\Gamma(t)$ of $\Omega(t)$ (if $\phi(\cdot, t)$ vanishes only on a set of zero Lebesgue measure) is then given by the zero level set, i.e.,

$$
\Gamma(t)=\{\phi(\cdot, t)=0\} .
$$

If the evolution of the shape is determined by a flow $x(t)=\xi(t, x(0))$ such that

$$
\frac{\mathrm{d} x}{\mathrm{~d} t}(t)=V(x(t), t),
$$

then the corresponding level set function $\phi$ is determined by the first-order Hamilton-Jacobi equation

$$
\frac{\partial \phi}{\partial t}+V \cdot \nabla \phi=0 \quad \text { in } \mathbb{R}^{N} \times \mathbb{R}^{+} .
$$

In the particular case of a velocity in normal direction, i.e.,

$$
V=v n \quad \text { on } \Gamma \times \mathbb{R}^{+},
$$

where $v$ is a scalar function and $n$ represents the unit outer normal on $\Gamma$, we can use the relation $n=\nabla \phi /|\nabla \phi|$ to compute evolution of the level set function from the nonlinear level set equation

$$
\frac{\partial \phi}{\partial t}+v|\nabla \phi|=0 \quad \text { in } \mathbb{R}^{N} \times \mathbb{R}^{+},
$$

where $v$ has to be extended also to $\mathbb{R}^{N}-\Gamma$. In general, evolutions with the same normal component of the velocity coincide (tangential components correspond to reparameterizations only), so that we will restrict our attention to the case (1.6).

\subsection{Level set methods for inverse problems and optimization}

In the following we review some recent developments in the construction of level set methods for inverse problems dealing with the reconstruction of shapes. The first and fundamental step towards 
level set methods for general classes of inverse problems was made by Santosa [66] in the case when a least-squares functional of the form

$$
J(\Omega)=\frac{1}{2}\left\|G\left(\chi_{\Omega}\right)-z\right\|^{2}
$$

is to be minimized, where $G$ is a nonlinear operator acting between $L^{2}(\Omega)$ and some Hilbert space $X$. By formal calculus of shape derivatives, Santosa deduced that

$$
J^{\prime}(\Omega) v=\int_{\Gamma} v\left(G^{\prime}\left(\chi_{\Omega}\right)^{*}\left(G\left(\chi_{\Omega}\right)-z\right)\right) \mathrm{d} s
$$

where $G^{\prime}\left(\chi_{\Omega}\right)^{*}: X \rightarrow L^{2}(\Theta)$ is the adjoint of the Fréchet derivative $G^{\prime}$ in $L^{2}(\Theta)$. We mention that in typical applications to inverse problems, $G^{\prime}\left(\chi_{\Omega}\right)^{*}$ is a smoothing operator, and hence the evaluation of the $L^{2}$-adjoint on the curve $\Gamma$ is well defined.

In order to obtain an evolution in descent direction Santosa now proposed to choose the velocity via

$$
v=-\left.G^{\prime}\left(\chi_{\Omega}\right)^{*}\left(G\left(\chi_{\Omega}\right)-z\right)\right|_{\Gamma} \quad \text { on } \Gamma
$$

where $\left.\varphi\right|_{\Gamma}$ denotes the restriction of a function defined on $\Theta$ to the curve $\Gamma \subset \Theta$. Santosa's approach seems to be related to a continuous version of the steepest descent algorithm, and to the method of asymptotic regularization for inverse problems (cf. [74]), where the evolution of an $L^{2}$-function towards a solution of $G(f)=z$ would be determined by

$$
\frac{\mathrm{d} f}{\mathrm{~d} t}=-G^{\prime}(f)^{*}(G(f)-z) .
$$

The restriction to shape derivatives and the choice of a normal velocity using only the information contained in the right-hand side of (1.9) may be dangerous for general operators $G$, since the corresponding evolution of the shape $\Omega(t)$ might stop at a shape which is no solution of $G\left(\chi_{\Omega}\right)=z$. In this paper, however, we will be concerned with the construction of level set methods using shape derivatives, a choice motivated by several reasons: First of all, there seems to be no way to generalize the approach in [18] to more general obstacle problems, which depend also on other geometric quantities than the indicator function of $\Omega$. Secondly, for all model problems we have in mind, the above problem will not appear, i.e., the evolution can stop at a solution only, which we shall show below. A third reason is that the numerical implementation of the method in [18] is less efficient than approaches like Santosa's, since some elliptic partial differential equations have to be solved to compute the velocity.

The approach by Santosa can be applied to more general shape reconstruction and optimization problems, by using the so-called speed or velocity method (cf. e.g. [26, 72, 77]) based on the shape derivative of the objective functional $J(\Omega)$. Shape derivatives are an important tool for shape optimization and reconstruction problems. Their underlying idea is to compute the variation of a functional $J(\Omega)$ when the shape is perturbed in normal direction with speed $v$. The corresponding shape derivative is then denoted by $J^{\prime}(\Omega) v$; for a comprehensive introduction to this topic and further details we refer to the monograph by Sokołowski and Zolésio [72] and to [55].

This derivative has (for a rather general class of problems, cf. [72]) a representation of the form

$$
J^{\prime}(\Omega) v=\int_{\Gamma} \rho v \mathrm{~d} s,
$$


where $\rho: \Gamma \rightarrow \mathbb{R}$ can depend on the solutions of the direct and some adjoint problems as well as on the geometry of the interface $\Gamma$ (e.g. via its curvature). In this case, one can choose the normal velocity on $\Gamma$ equal to $-\rho$ and use an appropriate extension to the computational domain. This obviously yields an evolution in descent direction, which only stops if the shape derivative vanishes.

Another desirable property for a level set method used to reconstruct interior obstacles such as in our introductory example is that the evolving zero level set remains a subset of the domain $\Theta$. This is not guaranteed automatically by an approach like Santosa's, but also depends on the extension of the velocity outside the interface. The standard methods for constructing extension velocities are based on constant extension in normal direction of the level set (cf. [1]) and allow no control of the velocity on the boundary. An alternative way to construct extension velocities is to choose $v=0$ on the boundary of the exterior domain (which should equal a level set for some positive value) and to extend $v$ e.g. via solving a Dirichlet problem for the Laplace equation on the exterior domain. For this extension, the boundary of the exterior domain remains equal to a positive level set during the evolution, and hence the zero level set must stay inside. As we shall see below, this extension is natural for one of the methods we propose.

\section{Elliptic model problems}

In the following we shall discuss some model inverse problems for elliptic equations, which serve as a motivation for our analysis and will be used subsequently as test examples. The basic guideline for choosing model problems is to give typical examples for shape optimization and shape reconstruction, representing a larger class of (more complicated) practical applications. For this sake we shall also give extensions of the problems and references to literature in the subsequent presentation.

EXAMPLE 1 (Support reconstruction) By the term support reconstruction we understand the type of problem considered by Santosa [66] and by the author in [18], where the shape enters into the inverse problem via its indicator function, i.e., the aim is to reconstruct the support of some function. Such problems typically appear in the identification of piecewise constant parameters in partial differential equations or in image processing. As an example for the reconstruction of an interior shape we consider one of the simplest problems appearing in practice, namely the identification of a domain $\Omega \subset \Theta \subset \mathbb{R}^{N}$ from $\left.u\right|_{M} \in L^{2}(M)$, where $u$ is the solution to

$$
-\Delta u=\chi_{\Omega} \quad \text { in } \Theta
$$

subject to homogeneous Dirichlet boundary conditions on $\Gamma_{d} \subset \partial \Theta$ and the Neumann condition

$$
\frac{\partial u}{\partial n}=g \quad \text { on } \Gamma_{n}=\partial \Theta-\Gamma_{d} .
$$

The set $M$ where the data are measured is either a subset of $\Gamma_{n}$ or of $\Theta$.

A prominent example in this class of problems is the inverse conductivity problem with one measurement, which means to identify $\Omega$ from the over-determined boundary value problem

$$
\begin{aligned}
-\operatorname{div}\left(\left(1+k \chi_{\Omega}\right) \nabla u\right) & =f & & \text { in } \Omega, \\
u & =g & & \text { on } \partial \Omega, \\
\frac{\partial u}{\partial n} & =h & & \text { on } \partial \Omega,
\end{aligned}
$$


for some positive real number $k$. Many aspects of this problem have been studied in the last decade, ranging from local identifiability properties (cf. [32, 42, 4]), over-size estimates (cf. [5, 6]) to the iterative solution and regularization (cf. [38,45,47]). In some cases also multiple Dirichletto-Neumann data $\left(g_{i}, h_{i}\right)$ are considered instead of the single measurement $(g, h)$; in other applications, (2.3) and (2.4) are used with a measurement of the state $u$ on $\Theta_{0} \subset \Theta-\Omega$. Both cases lead to global identifiability and stability results. A recent level-set based solution of the latter problem, using Santosa's strategy, has been discussed by Ito, Kunisch and Li [43].

The class of support reconstructions includes many examples where the jump set of parameters arising in partial differential equations is to be determined from indirect measurements. Such applications arise e.g. in inverse scattering (cf. [24] and the references therein), or in the characterization of semiconductor devices (cf. [20, 19]).

The associated least-squares functional is given by (with $f^{\delta}$ representing a noisy measurement)

$$
J(\Omega)=\frac{1}{2} \int_{M}\left|u-f^{\delta}\right|^{2} \mathrm{~d} s,
$$

with shape derivative

$$
J^{\prime}(\Omega) v=\int_{\Gamma} u^{*} v \mathrm{~d} s,
$$

where the adjoint $u^{*}$ satisfies

$$
\begin{aligned}
\Delta u^{*} & =0 & & \text { in } \Omega, \\
\frac{\partial u^{*}}{\partial n} & =\chi_{M}\left(u-f^{\delta}\right) & & \text { on } \Gamma_{n}, \\
u^{*} & =0 & & \text { on } \Gamma_{d} .
\end{aligned}
$$

in the case of boundary measurements $\left(M \subset \Gamma_{n}\right)$, and

$$
\begin{aligned}
-\Delta u^{*} & =\chi_{M}\left(u-f^{\delta}\right) & & \text { in } \Omega, \\
\frac{\partial u^{*}}{\partial n} & =0 & & \text { on } \Gamma_{n}, \\
u^{*} & =0 & & \text { on } \Gamma_{d} .
\end{aligned}
$$

in the case of distributed measurements $(M \subset \Omega)$.

For a detailed analysis of first and second shape derivatives for this problem and some further analysis we refer to Hettlich and Rundell [36].

Finally, we investigate the possible zeros of the shape derivative. If $J^{\prime}(\Omega) \equiv 0$ for some regular shape, i.e., $u^{*} \equiv 0$ on $\Gamma=\partial \Omega$, then by uniqueness for the Laplace equation we find that $u^{*} \equiv 0$ in $\Omega$. Hence, we also see that $u^{*}$ and $\partial u^{*} / \partial n$ vanish on $\Gamma$, and the uniqueness for the Cauchy problem implies that $u^{*} \equiv 0$ in $\Theta-\Omega$. Consequently, from the definition of $u^{*}$ we may conclude that $\left.u\right|_{M}=f^{\delta}$ on $M$, i.e., the residual is zero, too.

EXAMPLE 2 (Boundary and obstacle reconstruction) As boundary reconstruction problems or obstacle reconstruction, we summarize the class of inverse problems where the unknown is a part of the boundary of the domain on which some state equation has to be solved. In typical applications, this identification is based on overdetermination on a known part of the boundary, where Dirichlet and Neumann values of the solution are known. On the unknown part, usually a homogeneous 
boundary condition is specified. Our model problem for this class is again related to the Laplace equation: we consider the identification of $\Gamma=\partial \Omega$ from a measurement $u \in L^{2}(M)$, where $u$ is the solution of

$$
\begin{array}{rlrl}
\Delta u & =0 & & \text { in } \Theta-\Gamma, \\
\frac{\partial u}{\partial n}=g & & \text { on } \Gamma_{n}, \\
\frac{\partial u}{\partial n}=0 & & \text { on } \Gamma, \\
u=0 & & \text { on } \Gamma_{d} .
\end{array}
$$

The set $M$ where measurements are taken is either a subset of the fixed Neumann boundary $\Gamma_{n}$ or a subset of $\Theta$. Moreover, we assume that $g$ is not a constant function on $\Gamma_{n}$, which is needed for identifiability of $\Gamma$ (cf. [11] for further details on this problem). An analogous boundary reconstruction problem would consist in identifying an unaccessible boundary curve $\Gamma=\partial \Theta-\left(\Gamma_{n} \cap \Gamma_{d}\right)$ from measurements on the accessible part of the boundary.

This problem and variants with Dirichlet-type or mixed boundary conditions on the interface $\Gamma$ have been investigated recently with respect to identifiability and stability (cf. [3, 11, 13, 17]), which can be obtained using continuation techniques for elliptic Cauchy problems. The most important application of such a problem is inclusion detection in elastic media under anti-planar conditions (for the planar case one obtains the same problem with the Laplace equation replaced by the equations of linear elasticity, cf. [11]). The problem with Dirichlet condition on $\Gamma$ appears as an asymptotic case in identifying a p-n junction of a semiconductor device (cf. [20, 19]) and in corrosion detection from electrostatic or thermal data (cf. [46, 62, 73]). The parabolic variant of this problem is discussed by Bryan and Caudill [15, 16] as a model for thermal imaging. Park and Shin [59] discuss a similar identification problem with the Navier-Stokes system coupled to the heat equation as the underlying state equations, which is motivated by several applications such as thermal tomography or Brigdman crystal growth.

The shape derivative of the output least-squares functional $\left(f^{\delta}\right.$ representing the noisy data and $\left.M \subset \Gamma_{n}\right)$

$$
J(\Omega)=\tilde{J}(u, \Omega)=\frac{1}{2} \int_{M}\left|u-f^{\delta}\right|^{2} \mathrm{~d} s
$$

is given by

$$
J^{\prime}(\Omega) v=-\int_{\Gamma}\left[\nabla u . \nabla u^{*}\right] v \mathrm{~d} s,
$$

where [.] denotes the jump across $\Gamma$. The function $u^{*}$ solves the adjoint problem, which is given by

$$
\begin{aligned}
\Delta u^{*} & =0 & & \text { in } \Theta-\Gamma, \\
\frac{\partial u^{*}}{\partial n} & =\chi_{M}\left(u-f^{\delta}\right) & & \text { on } \Gamma_{n}, \\
\frac{\partial u^{*}}{\partial n} & =0 & & \text { on } \Gamma, \\
u^{*} & =0 & & \text { on } \Gamma_{d} .
\end{aligned}
$$

For further details on this problem and its shape derivative we refer to [11], where also penalization 
by perimeter, i.e., minimization of the functional

$$
J_{\alpha}(\Omega)=\frac{1}{2} \int_{M}\left|u-f^{\delta}\right|^{2} \mathrm{~d} s+\alpha \mathcal{H}^{1}(\Gamma)
$$

has been analyzed as a regularization method with respect to the Hausdorff metric. For the shape derivative, this additional regularization term would yield again a second term involving the integral over-curvature.

Note that the second model problem might also give some insight to typical problems in shape optimization, where usually a functional of the state is minimized subject to the same type of state equation and boundary conditions (with the Laplace equation being replaced by the elasticity system in structural optimization, cf. [12, 60, 72]). Level set methods for the classical minimum compliance problem (cf. [12]) have been used by Sethian and Wiegmann [70] as well as Allaire, Jouve and Toader [7], and produced good numerical results (however, without theoretical justification).

\section{A functional-analytic framework}

In this section we shall provide a functional-analytic basis for the construction of level set methods based on the idea of gradient flows for some energy functional. For convenience we start with a short review of gradient flows for general systems and their relations to optimization and regularization, and then carry these ideas over to gradient flows for geometric configurations. Finally, we give examples of the most important norms in which gradient flows can be derived.

\subsection{Gradient flows of the total energy}

Using standard ideas of equilibrium thermodynamics, many physical evolution models can be determined as gradient flows for the total energy $\mathcal{E}$ of a system. For example, the well-known heat equation

$$
\frac{\partial w}{\partial t}=\operatorname{div}(D \nabla w) \quad \text { in } \Omega \subset \mathbb{R}^{N}
$$

is determined as a gradient flow of the form

$$
\frac{\partial w}{\partial t}=-\nabla_{w} \mathcal{E}(w)
$$

for the total energy

$$
\mathcal{E}(w)=\frac{1}{2} \int_{\Omega} D(x)|\nabla w(x)|^{2} \mathrm{~d} x .
$$

If the aim is to derive evolution models for geometric configurations instead of functions, the form (3.1) is not directly applicable, since one has to give a meaning to the term on the left-hand side describing the evolution of the geometry as well as to the variation of the energy on the right-hand side. The latter can be realized in the framework of shape derivatives, which gives the variation of the energy for variations of the domain in normal direction. For the first, we need a different formulation of gradient flows. For this sake we return to the simple example of the heat equation. Following the 
presentation by Almgren and Taylor [8], we consider the following variational problem for a small time step $\Delta t$ :

$$
\frac{1}{2} \int_{\Omega} D(x)|\nabla w(x, t+\Delta t)|^{2} \mathrm{~d} x+\frac{1}{2 \Delta t} \int_{\Omega}|w(x, t+\Delta t)-w(x, t)|^{2} \mathrm{~d} x \rightarrow \min _{w(\cdot, t+\Delta t) \in \mathcal{W}},
$$

where $\mathcal{W}$ represents an appropriate set of admissible solutions (e.g. $H_{0}^{1}(\Omega)$ ). From the first-order optimality conditions one finds that the solution of this variational problem satisfies (at least in the standard weak sense)

$$
-\operatorname{div}(D(x) \nabla w(x, t+\Delta t))+\frac{w(x, t+\Delta t)-w(x, t)}{\Delta t}=0,
$$

which leads to the heat equation as $\Delta t \rightarrow 0$. The general approach corresponding to (3.2) is given by the variational problem

$$
\mathcal{E}(w(\cdot, t+\Delta t))+\frac{1}{2 \Delta t}\|w(\cdot, t+\Delta t)-w(\cdot, t)\|^{2} \rightarrow \min _{w(\cdot, t+\Delta t) \in \mathcal{W}}
$$

and the corresponding first-order optimality condition leads to the gradient flow as $\Delta t \rightarrow 0$.

It is worth noting that gradient flows have a long tradition in the regularization of inverse problems. Of particular importance is the method of asymptotic regularization ([29]). For a linear operator equation of the form $A w=f$, with $A: \mathcal{W} \rightarrow \mathcal{Z}$ being an operator acting between Hilbert spaces, one can define the energy as the corresponding least-squares functional

$$
\mathcal{E}(w)=\frac{1}{2}\|A w-f\|^{2}
$$

and asymptotic regularization as the corresponding gradient flow is given by

$$
\frac{\partial w}{\partial t}=-A^{*}(A w-f)
$$

where $A^{*}: \mathcal{Z} \rightarrow \mathcal{W}$ is the adjoint operator. In the case of a nonlinear operator $A$ analogous reasoning leads to

$$
\frac{\partial w}{\partial t}=-A^{\prime}(w)^{*}(A(w)-f)
$$

where $A^{\prime}(w)$ denotes the Fréchet derivative of $A$ at $w \in \mathcal{W}$. For an analysis of the method of asymptotic regularization in the linear case and a detailed discussion in a general framework for ill-posed problems we refer to Chapter 4 of the monograph by Engl et al. [29], and for the analysis in the nonlinear case to Tautenhahn [74]. Moreover, this method has been applied successfully to ill-posed problems of different origin, such as parameter identification (cf. [39,40]).

From the method of asymptotic regularization one can deduce a variety of well-known regularization schemes by specific time discretizations. For example, an explicit time discretization yields the so-called Landweber iteration (cf. [29,48] for the linear and [14, 35, 67] for the nonlinear case)

$$
w_{k+1}=w_{k}-\Delta t A^{\prime}\left(w_{k}\right)^{*}\left(A\left(w_{k}\right)-f\right), \quad k=0,1, \ldots
$$


Another important scheme, the method of iterated Tikhonov regularization (cf. [29, 33, 34]), consisting of the sequence of minimization problems

$$
\frac{1}{2}\left\|A\left(w_{k+1}\right)-f\right\|^{2}+\frac{\alpha}{2}\left\|w_{k+1}-w_{k}\right\|^{2} \rightarrow \min _{w_{k+1} \in \mathcal{W}}, \quad k=0,1, \ldots,
$$

is obtained by an implicit time discretization with $\Delta t=\alpha^{-1}$. One observes that this variational problem coincides (apart from different notations) with the one used in the approach by Almgren and Taylor presented above, which provides further arguments for our idea that many schemes arising from variational models in physics can also serve as good regularization and optimization schemes. Motivated by this analogy, in the following sections we shall therefore try to carry over the models for evolution towards optimal geometries in materials science to evolution methods for shape optimization and reconstruction. The level set method will provide a natural representation of evolving shapes in this context.

Before proceeding in the announced direction, we mention some further regularization approaches based on gradient flows. An analogous reasoning has been used by Scherzer and Weickert [68] in the context of mathematical imaging to obtain relations between diffusion filtering and generalizations of iterated Tikhonov regularization. More precisely they showed that diffusion filters of the form

$$
\frac{\partial w}{\partial t}=\operatorname{div}\left(g\left(|\nabla w|^{2}\right) \nabla w\right) \quad \text { in } \Omega \times \mathbb{R}^{+}
$$

with the degraded image as initial value correspond to the limit of the generalized iterated Tikhonov regularization method

$$
\int_{\Omega} G\left(|\nabla w(x, t+\Delta t)|^{2}\right) \mathrm{d} x+\frac{1}{2 \Delta t} \int_{\Omega}|w(x, t+\Delta t)-w(x, t)|^{2} \mathrm{~d} x \rightarrow \min _{w(\cdot, t+\Delta t) \in \mathcal{W}},
$$

with $G$ being the antiderivative of the filter function $g$. The work by Scherzer and Weickert [68] and subsequent investigations in [61] demonstrate the suitability of gradient flows for the regularization of inverse problems in a rather general framework.

\subsection{Gradient flows for geometric configurations}

If we consider instead of functions the evolution of a curve $\Gamma$ with associated energy $\mathcal{E}(\Gamma)$, then we can define the variations of $\Gamma$ due to a field $v$ in normal directions (as in the setup for shape derivatives) given by

$$
\Gamma^{v}(t+\Delta t):=\{x+\Delta t v(x) n(x) \mid x \in \Gamma(t)\}
$$

and try to obtain a gradient flow from the minimization problem

$$
\mathcal{E}\left(\Gamma^{v}(t+\Delta t)\right)+\frac{\Delta t}{2}\|v\|_{\mathcal{V}}^{2} \rightarrow \min _{v \in \mathcal{V}}
$$

where $\mathcal{V}$ is a Hilbert space of suitable domain variations and $\Delta t\|v\|$ measures the variation between $\Gamma(t)$ and $\gamma^{v}(t+\Delta t)$. The solution of this minimization problem satisfies the first-order optimality condition

$$
\frac{\partial \mathcal{E}}{\partial v}\left(\Gamma^{v}(t+\Delta t)\right) \Delta t w+\Delta t\langle v, w\rangle \mathcal{V}=0, \quad \forall w \in \mathcal{V} .
$$


Division by $\Delta t$ and letting $\Delta t \rightarrow 0$ finally yields

$$
\mathcal{E}^{\prime}\left(\Gamma^{v}(t+\Delta t)\right) w+\langle v, w\rangle_{\mathcal{V}}=0, \quad \forall w \in \mathcal{V}
$$

where $\mathcal{E}^{\prime}\left(\Gamma^{v}(t+\Delta t)\right) w$ denotes the shape derivative of $\mathcal{E}$ with respect to the normal variation $w$. If the shape derivative exists and defines a continuous linear form on $\mathcal{V}$, then the Riesz representation theorem guarantees the existence and uniqueness of a variation $v \in \mathcal{V}$ satisfying (3.14).

We finally note that the above definition can be extended to a level set framework by defining $\Gamma^{v}(t+\Delta t)$ not via $[3.11$, but as the zero level set of $\phi(\cdot, t+\Delta t)$, where $\phi$ is a solution of the level set equation (1.7) in the time interval $(t, t+\Delta t)$, with $\Gamma(t)=\{\phi(\cdot, t)=0\}$. A canonical choice $\phi(\cdot, t)$ seems to be the signed distance function to $\Gamma(t)$, which can also be used to compute shape derivatives and to define metrics on shapes (cf. [10,25]).

\subsection{Examples of inner products for the shape variation}

In the following we present the probably most important possibilities for Hilbert space norms (and corresponding inner products) of shape variations and derive the corresponding form of 3.14 . We will divide the norms into five types (with different norms within one type being equivalent), representing the values $r=1, \frac{1}{2}, 0,-\frac{1}{2}, 1$ in the scale of Sobolev spaces $H^{r}(\Gamma)$. The inner products for integer $s$ have been discussed as models for microstructural evolution (cf. [21, 75]), but for inverse problems also noninteger values of $r$ seem to be of interest. It turns out that each of the discussed inner poducts (and corresponding norms) has special properties and might yield advantages for certain problem classes.

In the following we will assume that there exists a function $\rho: \Gamma \rightarrow \mathbb{R}$ such that the shape derivative can be written as

$$
\mathcal{E}^{\prime}(\Gamma) w=\int_{\Gamma} \rho(x) w(x) \mathrm{d} s(x), \quad \forall w \in \mathcal{V} .
$$

As we have seen above, this assumption is not restrictive for the types of problems we have in mind, and holds in great generality (cf. [25,72])—a result sometimes called the Hadamard-Zolésio structure theorem.

\section{$H^{1}$-norm: "Laplace-Beltrami flow"}

As a first possibility for choosing a Hilbert space norm on $\Gamma$ we investigate the space $\mathcal{V}=H^{1}(\Gamma)$ with the inner product

$$
\langle v, w\rangle_{H^{1}(\Gamma)}=\int_{\Gamma}\left(\frac{\partial v}{\partial s} \frac{\partial w}{\partial s}+v w\right) \mathrm{d} s, \quad \forall v, w \in H^{1}(\Gamma),
$$

where $s$ is the arclength variable. This choice is suitable if $\Gamma$ consists of connected curves and leads to the partial differential equation

$$
-\frac{\partial^{2} v}{\partial s^{2}}+v=-\rho \quad \text { on } \Gamma
$$


for the variation $v$ defined by (3.14), involving the so-called Laplace-Beltrami operator. If a connected component of $\Gamma$ is nonclosed, then this equation has to be supplemented by the Neumann type boundary condition

$$
\frac{\partial v}{\partial s}=0 \quad \text { on } \partial \Gamma .
$$

An obvious generalization of the standard inner product in $H^{1}$ is a weighted one, i.e.,

$$
\langle v, w\rangle_{H_{M}^{1}(\Gamma)}=\int_{\Gamma}\left(M_{1} \frac{\partial v}{\partial s} \frac{\partial w}{\partial s}+M_{0} v w\right) \mathrm{d} s, \quad \forall v, w \in H^{1}(\Gamma),
$$

for some positive function $M_{i}: \Gamma \rightarrow \mathbb{R}, i=0,1$. In this case we obtain the anisotropic elliptic equation

$$
-\frac{\partial}{\partial s}\left(M_{1} \frac{\partial v}{\partial s}\right)+M_{0} v=-\rho \quad \text { on } \Gamma .
$$

In some applications, variants of the $H^{1}$ inner product 3.16 corresponding to subspaces are of importance. A first important example arises from problems where the volume of $\Omega$ shall be conserved during the evolution. The appropriate subspace of variations in $H^{1}(\Gamma)$ is given by

$$
H_{\diamond}^{1}(\Gamma)=\left\{v \in H^{1}(\Gamma) \mid \int_{\Gamma} v \mathrm{~d} s=0\right\},
$$

which incorporates the volume conservation. A simple inner product on this space of variations is given by

$$
\langle v, w\rangle_{H_{\diamond}^{1}(\Gamma)}=\int_{\Gamma} \frac{\partial v}{\partial s} \frac{\partial w}{\partial s} \mathrm{~d} s, \quad \forall v, w \in H_{\diamond}^{1}(\Gamma),
$$

and it defines a norm equivalent to the original $H^{1}$-norm because of the Poincare inequality. The partial differential equation for 3.14 is

$$
\frac{\partial^{2} v}{\partial s^{2}}=\rho \quad \text { on } \Gamma
$$

supplemented by the integral condition $\int_{\Gamma} v \mathrm{~d} s=0$ and again homogeneous Neumann boundary conditions on $\partial \Gamma$.

Another important subspace is

$$
H_{0}^{1}(\Gamma)=\left\{v \in H^{1}(\Gamma)|v|_{\partial \Gamma}=0\right\}
$$

with the same inner product as $H_{\diamond}^{1}(\Gamma)$. Since variations of this type keep the boundary points of $\Gamma$ fixed, they are of particular interest for shape reconstruction or optimization problems where the unknown $\Gamma$ is a nonclosed curve whose boundary points are known. In this case, 3.14 results again in the equation 3.23, but now supplemented by the Dirichlet boundary condition $v=0$ on $\partial \Gamma$.

$H^{1 / 2}$-norm: "Stefan-like flow"

Sobolev spaces of fractional order (cf. [49,65]) such as $H^{1 / 2}(\Gamma)$ are much more difficult to define and to handle than integer-order spaces such as $H^{1}(\Gamma)$ or $H^{0}(\Gamma)=L^{2}(\Gamma)$. Although not used 
much in this context, the space $H^{1 / 2}(\Gamma)$ seems to be of interest for our purpose, since its elements are just the Dirichlet values on $\Gamma$ of an $H^{1}$-function in $\Omega, \Theta-\Omega$ or $\Theta-\Gamma$. The space $H^{1 / 2}(\Gamma)$ has been used successfully by Hettlich and Rundell [37] to construct iterative regularization methods for the identification of the support of a source term in an elliptic partial differential equation. However, their iterative solution methods were based again on parameterizations of the boundary curve $\Gamma$ and limited to simply connected domains $\Omega$.

In general, the space $H^{1 / 2}(\Gamma)$ and its inner product are defined by the Hilbert space interpolation of $H^{1}(\Gamma)$ and $L^{2}(\Gamma)$ (cf. Lions and Magenes [49] for a comprehensive treatment of this topic), which is unfortunately not very useful for practical computations. An alternative definition of inner products on $H^{1 / 2}(\Gamma)$ (leading to equivalent norms) is possible via the characterization of its elements as traces of elements in $H^{1}(\Omega)$. E.g., if $\Gamma=\partial \Omega$ consists of closed components only, then we can define an inner product via

$$
\langle v, w\rangle_{H^{1 / 2}(\Gamma)}=\int_{\Omega}(\nabla \tilde{v} . \nabla \tilde{w}) \mathrm{d} x, \quad \forall v, w \in H^{1 / 2}(\Gamma),
$$

where $\tilde{v} \in H^{1}(\Omega)$ and $\tilde{w} \in H^{1}(\Omega)$ are the unique extensions of $v$ and $w$, respectively, to $\Omega$, satisfying

$$
\Delta \tilde{v}=\Delta \tilde{w}=0 \quad \text { in } \Omega .
$$

An application of Gauss' Theorem for sufficiently regular functions $v$ and $w$ shows that

$$
\langle v, w\rangle_{H^{1 / 2}(\Gamma)}=\int_{\Omega}(\nabla \tilde{v} . \nabla \tilde{w}) \mathrm{d} x=\int_{\Gamma} \frac{\partial v}{\partial n} w \mathrm{~d} s,
$$

and hence (3.14) is just the weak form of the Neumann-type condition

$$
\frac{\partial v}{\partial n}=-\rho \quad \text { on } \Gamma .
$$

If $\Omega$ is in the interior of an outer domain $\Theta$, then we can also define an equivalent norm by (3.25) with $\tilde{v} \in H^{1}(\Theta-\Omega)$ and $\tilde{w} \in H^{1}(\Theta-\Omega)$ being the extensions solving the Laplace equation in $\Theta-\Omega$ and satisfying an additional boundary condition on $\partial \Theta$. This additional boundary condition allows one to incorporate further a priori information such as $\Omega \subset \Theta$ into a level set evolution. If we choose $\Theta$ as a level set of the initial level set function $\phi_{0}$, and use the boundary condition $\tilde{v}=0$ on $\partial \Theta$, then the level set $\Theta$ will be unchanged during the evolution, and hence the zero level set $\Omega$ remains a subset.

In the last case as well as if $\Gamma$ is an interface in $\Theta$, we have another possibility to define an equivalent $H^{1 / 2}$-norm, namely as the $H^{1}$-norm of an extension $\tilde{v}$ to $\Theta-\Gamma$, i.e.,

$$
\langle v, w\rangle_{H^{1 / 2}(\Gamma)}=\int_{\Theta-\Gamma}(\nabla \tilde{v} . \nabla \tilde{w}) \mathrm{d} x, \quad \forall v, w \in H^{1 / 2}(\Gamma),
$$

where $\tilde{v}, \tilde{w} \in H^{1}(\Theta-\Gamma)$ are extensions of $v$ and $w$ satisfying the homogeneous Laplace equation on $\Theta-\Gamma$ and an additional boundary condition on $\partial \Theta$ (e.g. a homogeneous Dirichlet condition). With this choice of the inner product, (3.14) becomes the weak formulation of the homogeneous Laplace equation in $\Theta-\Gamma$ (for the extension $\tilde{v}$ ) with jump condition $[\partial v / \partial n]=-\rho$ on $\Gamma$.

We finally want to mention that in all choices of inner products for the $H^{1 / 2}$-norm the computation of an extension velocity $\tilde{v}$ is automatically included in the choice of the velocity via 
(3.14). This is a particular advantage in connection with level set methods, since they need an extension velocity in a larger domain like $\Theta$. Moreover, we have seen that by choosing appropriate boundary conditions on $\Theta$ and a suitable initial value for the level set function, we can obtain an evolution of the zero level set that stays inside $\Gamma$, which is important in many applications.

\section{$L^{2}$-norm: "Hadamard flow"}

Possibly the simplest inner product is the one of variations in $L^{2}(\Gamma)$, given by

$$
\langle v, w\rangle_{L^{2}(\Gamma)}=\int_{\Gamma} v w \mathrm{~d} s, \quad \forall v, w \in L^{2}(\Gamma) .
$$

In this case, 3.14 results in the explicit formula

$$
v=-\rho \quad \text { on } \Gamma \text {, }
$$

which coincides with Santosa's method of choosing the normal velocity and the classical version of the speed method in shape optimization.

An anisotropic version of the $L^{2}$ inner product is given by

$$
\langle v, w\rangle_{L^{2}(\Gamma)}=\int_{\Gamma} \frac{v w}{M} \mathrm{~d} s, \quad \forall v, w \in L^{2}(\Gamma),
$$

with a bounded function $M: \Gamma \rightarrow \mathbb{R}^{+}$. The formula for $v$ obtained from 3.14 is then given by

$$
v=-M \rho \quad \text { on } \Gamma \text {. }
$$

Anisotropic functionals are of particular interest if the $M$ is a function of the outer normal on $\Gamma$, i.e., $M=\tilde{M}(n)$, where $\tilde{M}: S^{1} \rightarrow \mathbb{R}_{+}$and $S^{1}$ being the unit sphere in $\mathbb{R}^{2}$. In materials science, anisotropies of this kind are introduced in order to model the structure of crystal lattices, and consequently they are often nonsmooth functions. For shape optimization such an anisotropic penalty term might be used to achieve minimizers that prefer certain geometric structures.

\section{$H^{-1 / 2}$-norm: "Mullins-Sekerka flow”}

In a similar way to $H^{1 / 2}(\Gamma)$ we can characterize $H^{-1 / 2}(\Gamma)$ as the space of Neumann values on $\Gamma$ of functions in $H^{1}(\Omega)$. For constructing an extension of the Neumann values, one has to be more careful, since the Laplace equation with general Neumann boundary conditions might have no solution. A solution to $\Delta \psi=0$ in $\Omega$ supplemented by $\partial \psi / \partial n=v$ on $\Gamma=\partial \Omega$ exists if and only if $\int_{\Gamma} v \mathrm{~d} s=0$. In the level set context, such a choice for the velocity implies that the volume is conserved during the evolution, which is desirable for certain applications. If there is no volume constraint, then we have to use a more general equation like $-\Delta \psi+\psi=0$ combined with the Neumann boundary condition. For simplicity, we will not consider the latter case, but the results in this case are analogous.

For $\int_{\Gamma} v \mathrm{~d} s=0$, the Neumann problem

$$
\begin{aligned}
-\Delta \psi_{v}=0 & \text { in } \Omega, \\
\frac{\partial \psi_{v}}{\partial n}=v & \text { on } \partial \Omega,
\end{aligned}
$$


has a unique solution in the space

$$
H_{\diamond}^{1}(\Omega)=\left\{\psi \in H^{1}(\Omega) \mid \int_{\Gamma} \psi \mathrm{d} x=0\right\} .
$$

The above equation for $\psi_{v}$ leads in a natural way to the inner product

$$
\langle v, w\rangle_{H^{1 / 2}(\Gamma)}=\int_{\Omega}\left(\nabla \psi_{v} . \nabla \psi_{w}\right) \mathrm{d} x, \quad \forall v, w \in H^{1 / 2}(\Gamma),
$$

where $\psi_{v}$ and $\psi_{w}$ are the solutions in $H_{\diamond}^{1}(\Omega)$ of 3.33, 3.34 with Neumann values $v$ and $w$, respectively.

Using (3.14), $\int_{\Gamma} w \mathrm{~d} s=0$, and Gauss' Theorem we obtain

$$
-\int_{\Gamma}(\rho-\bar{\rho}) w \mathrm{~d} s=-\int_{\Gamma} \rho w \mathrm{~d} s=\int_{\Omega} \psi_{v} \psi_{w} \mathrm{~d} x=\int_{\Gamma} \psi_{v} w \mathrm{~d} s
$$

with $\mathcal{H}^{1}(\Gamma) \bar{\rho}=\int_{\Gamma} \rho$ ds. Hence, we obtain $\psi_{v}=-\rho+\bar{\rho}$ on $\Gamma$ and $\Delta \psi_{v}=0$ in $\Omega$. Due to

$$
\int_{\Gamma} v \mathrm{~d} s=\int_{\Gamma}(-\rho+\bar{\rho}) \mathrm{d} s=0,
$$

the resulting function $\psi_{v}$ is an element of $H_{\diamond}^{1}(\Omega)$ and $v=\partial \psi_{v} / \partial n$ equals its Neumann value on $\Gamma$. Not surprisingly, the computation of $v$ in this case is somehow dual to the computation for the $H^{1 / 2}$-norm, where $v$ was chosen as the Dirichlet value of a solution of the Laplace equation with Neumann values equal to $-\rho$.

The evolution obtained in the case of $H^{-1 / 2}(\Gamma)$ has the physical interpretation of motion by bulk diffusion; it is called Mullins-Sekerka flow (cf. [54,51]) in the materials science community or also Hele-Shaw flow (cf. e.g. [41] and the references therein).

We finally mention that instead of $\int_{\Gamma} \psi \mathrm{d} x=0$ in the definition of $H_{\diamond}^{1}(\Omega)$ one could use $\ell(\psi)=0$, with $\ell$ being a continuous linear functional on $H^{1}(\Omega)$ not vanishing on the subspace of continuous functions, for the definition of a space $H_{\diamond}^{1}(\Omega)$ still providing a unique solution. However, a different choice of the functional $\ell$ would not guarantee that the solution of the homogeneous Laplace equation with Dirichlet values depending on $\rho$ satisfies the integral condition, and hence one cannot show that a corresponding Neumann value $v$ exists.

$H^{-1}$-norm: "surface diffusion flow"

A rather weak norm, but nonetheless a very important one in materials science is the $H^{-1}$-norm, which is defined on the space

$$
H^{-1}(\Gamma)=\left\{v \in \mathcal{D}^{\prime}(\Gamma) \mid \exists \omega_{v} \in H_{0}^{1}(\Gamma): \frac{\partial^{2} \omega_{v}}{\partial s^{2}}=v\right\},
$$

and generated by the inner product

$$
\langle v, w\rangle_{H^{-1}(\Gamma)}=\int_{\Gamma} \frac{\partial \omega_{v}}{\partial s} \frac{\partial \omega_{w}}{\partial s} \mathrm{~d} s, \quad \forall v, w \in H^{-1}(\Gamma),
$$


with $\omega_{v}$ and $\omega_{w}$ being the $H_{0}^{1}$-functions associated to $v$ and $w$, respectively, via the Laplace equation. An application of Gauss' Theorem shows that 3.14 leads to $\omega_{w}=\rho$ on $\Gamma$, and hence the velocity is given by

$$
v=\frac{\partial^{2} \rho}{\partial s^{2}} \quad \text { on } \Gamma .
$$

An evolution of this type is often called surface diffusion (cf. e.g. [30]); if the energy coincides with the perimeter of $\Gamma$, then also the term motion by Laplacian of curvature is used (note that $\rho$ equals the curvature in this case, and thus $v$ is the surface Laplacian of the curvature, cf. e.g. [9, 23]). When interpreted as a partial differential equation for the curve, the motion by Laplacian of curvature is fourth-order parabolic, and hence its numerical approximation is a difficult task. In particular, explicit time discretizations lead to extremely small time steps (proportional to the fourth order of the fineness of the spatial discretization) in order to achieve stability, while fully implicit schemes yield very stiff problems, at least in the case of the original surface diffusion flow (cf. [23, 71]).

\section{Application to the model problems}

In this section we apply the methods deduced above to our model problems. We discuss the possible choices of norms for each example and discuss its particular properties. In addition, we give the results of some numerical experiments to test the behavior of the resulting level set methods. For all numerical examples we use an explicit weighted essentially nonoscillatory scheme for the level set equation (cf. [44] for details), while we solve the direct and adjoint problem with the finite element method using a fictitious domain approach, which depends on the problem and will therefore be outlined below.

\subsection{Support reconstruction}

For the problem of support reconstruction in Example 1 we have several possibilities for the choice of the norm, the weakest being $H^{-1 / 2}(\Gamma)$, since the shape derivative (2.7) is a continuous functional on this space (when interpreted as a linear functional of the normal velocity $v$, cf. [36]). A particular case yielding further insight into the problem is that the volume of $\Omega$ is known in advance and equals the volume of the initial value $\Omega(0)$, and that $M \cap \Omega(t)=\emptyset$. In this case we may choose a volume-conserving flow, and revisiting the construction of such a flow via a solution $\psi$ of the Laplace equation in $\Omega$, we observe that the adjoint solution $u^{*}$ satisfies

$$
\begin{aligned}
\Delta\left(\psi-u^{*}\right)=0 & \text { in } \Omega, \\
\psi-u^{*}=0 & \text { on } \Gamma .
\end{aligned}
$$

This implies $\psi \equiv u^{*}$ by a uniqueness result for the Laplace equation in $\Omega$, since $\psi=u^{*}$ on $\partial \Omega$. Hence, the choice of the velocity corresponding to a gradient flow in $H^{-1 / 2}(\Gamma)$ is just

$$
v=-\frac{\partial u^{*}}{\partial n} \quad \text { on } \Gamma,
$$

so that we need not solve an additional boundary value problem in $\Omega$. For the norm of $L^{2}(\Gamma)$, the velocity is simply determined as $v=-u^{*}$, while for the case of a velocity in $H^{1 / 2}(\Gamma)$ we solve an additional partial differential equation as in the previous example, now with $[\partial \tilde{v} / \partial n]=u^{*}$ 
on $\Gamma$. Hence, in this case the three choices are related to different levels of derivatives of the adjoint solution $u^{*}$ : the Mullins-Sekerka flow to the first derivative, Santosa's flow to the derivative of order zero ( $u^{*}$ itself) and the Stefan-type flow to the solution of a Laplace equation, which can be interpreted as a generalized antiderivative (in spatial dimension one it equals exactly the antiderivative).

For numerical purposes, we approximate the indicator function $\chi_{\Omega}=H(\phi)$ by a continuous function $H_{\epsilon}(\phi)$, with $H_{\epsilon}$ being continuously differentiable and approximating the Heaviside function $H$ as $\epsilon \rightarrow 0$. For our purpose, there are several advantages of this relaxation: First of all, the solution with smoothed source is more regular, and thus the convergence rate of a finite element method (as the discretization fineness tends to zero) is higher. Of course, there is an additional error in terms of $\epsilon$ introduced by the relaxation, but with an appropriate relation between $\epsilon$ and the discretization fineness, good results can be achieved (cf. [76] for further details). A second advantage is that the shape derivative of the problem with relaxation is not an interface problem as for discontinuous source, but again an elliptic problem with a source dependent on $\phi$. The weak formulation of this derivative is given by

$$
\int_{\Theta} \nabla u^{\prime} \cdot \nabla w \mathrm{~d} x=-\int_{\Theta} H_{\epsilon}^{\prime}(\phi)|\nabla \phi| w \mathrm{~d} x .
$$

If we use again the adjoint solution $u^{*}$ (defined as above) and apply the coarea formula, we obtain

$$
\frac{\mathrm{d} J}{\mathrm{~d} t}=-\int_{\Theta} H_{\epsilon}^{\prime}(\phi)|\nabla \phi| v u^{*} \mathrm{~d} x=-\int_{\mathbb{R}} H_{\epsilon}^{\prime}(p) \int_{\{\phi=p\} \cap \Theta} v u^{*} \mathrm{~d} \mathcal{H}^{1} \mathrm{~d} p,
$$

from which we obtain the velocities for the different norms.

For a first numerical test, we investigate the problem in $\Theta=(-1,1)^{2}$ with homogeneous Dirichlet boundary conditions on $\partial \Theta$ and a distributed measurement of the state $u$ on $M=$ $(-1,1) \times(0.1,1)$. The exact solution of the inverse problem is the union of two small circles (shown in the pictures below) and the initial shape for the level set evolution is a single large circle. Data are generated in a synthetic way by solving the direct problem on a finer grid and interpolation to a fixed measurement grid in the set $M$. In addition, high-frequency noise of around 5\% is added to the exact solution before interpolation in order to avoid so-called inverse crimes (i.e., data and noise generation and solution of the inverse problem on the same grid).

We discretize the level set method on a regular grid of $128 \times 128$ points, with time step for an interval $(t, t+\Delta t)$ chosen to respect the CFL-condition for stability, i.e.,

$$
\Delta t \sup _{x}|v(x, t)|=0.9 \Delta x,
$$

where $\Delta x$ is the fineness of the spatial discretization. Since the time is artificial in our case and depends on the scaling of the velocities, we compare the methods with respect to the number of time steps (which can be interpreted as number of iterations) and not with respect to the value of the time variable. We plot the evolving shape at each 25-th time step from 25 to 150 in Figures 13.

Figure 1 shows the evolution according to Santosa's method (velocity norm in $L^{2}(\Gamma)$ ), Figure 2 according to the Mullins-Sekerka flow (velocity norm in $H^{-1 / 2}(\Gamma)$ ), and Figure 3 according to the Stefan-type flow (velocity norm in $H^{1 / 2}(\Gamma)$ ).

From a comparison of the plots one observes that the weakest possible norm for this problem, namely the Mullins-Sekerka flow, yields the fastest convergence towards the exact solution. For 

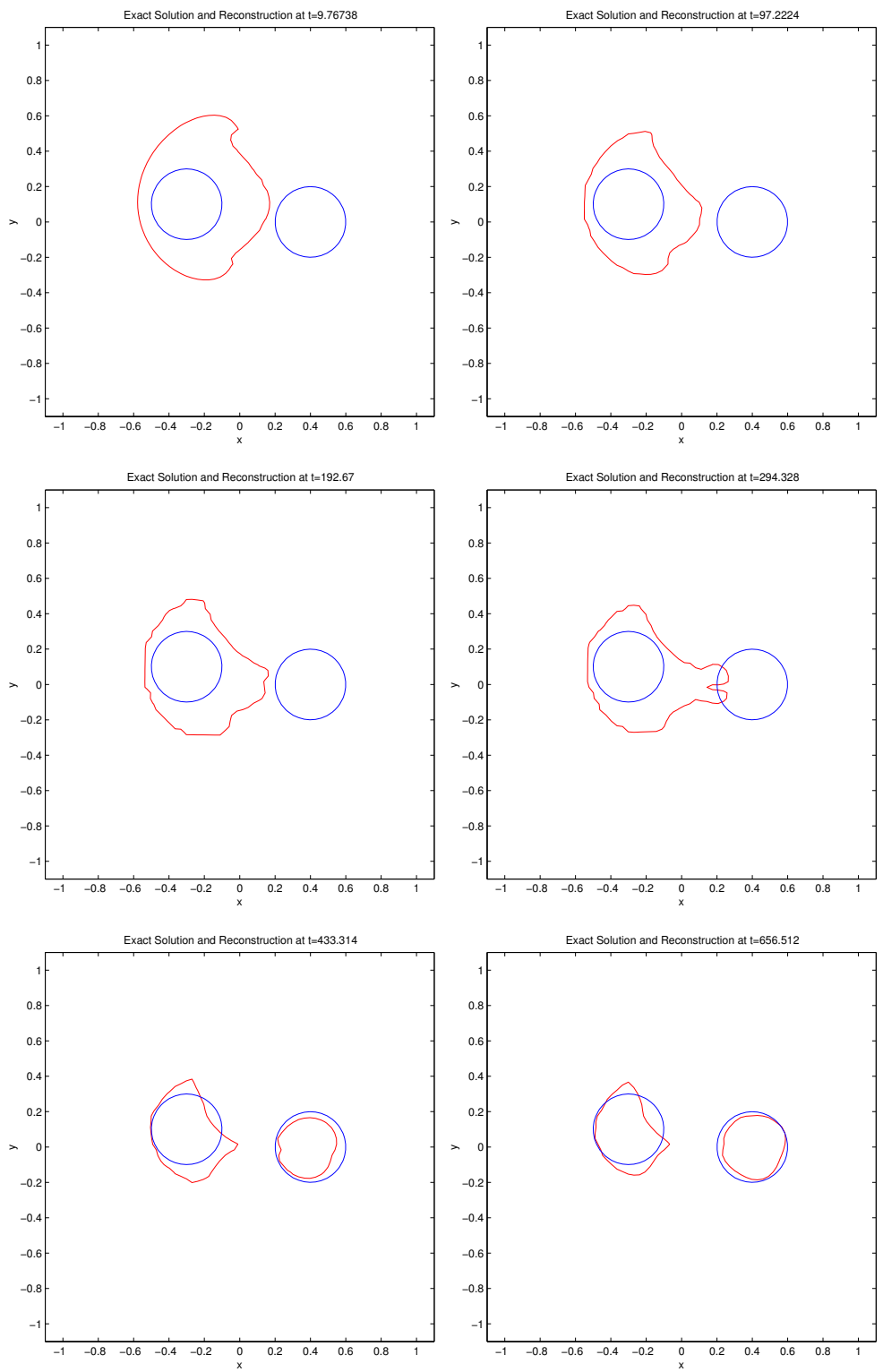

FIG. 1. Reconstructions with the Hadamard flow at iterations 25, 50, 75, 100, 125 and 150.

the standard approach in Figure 1 one observes convergence to a solution of the same quality as for the Mullins-Sekerka flow, while the Stefan-type flow shown in Figure 3 does not split the domain within the first 150 time steps. Our numerical experiments showed that this splitting occurs for the Stefan-type flow after more than 250 time steps and a similar convergence behavior is obtained afterwards. A quantitative comparison between the three different flows is given in Figure 4, where the evolution of the residuals (top) and of the $L^{1}$-error (bottom, starting 

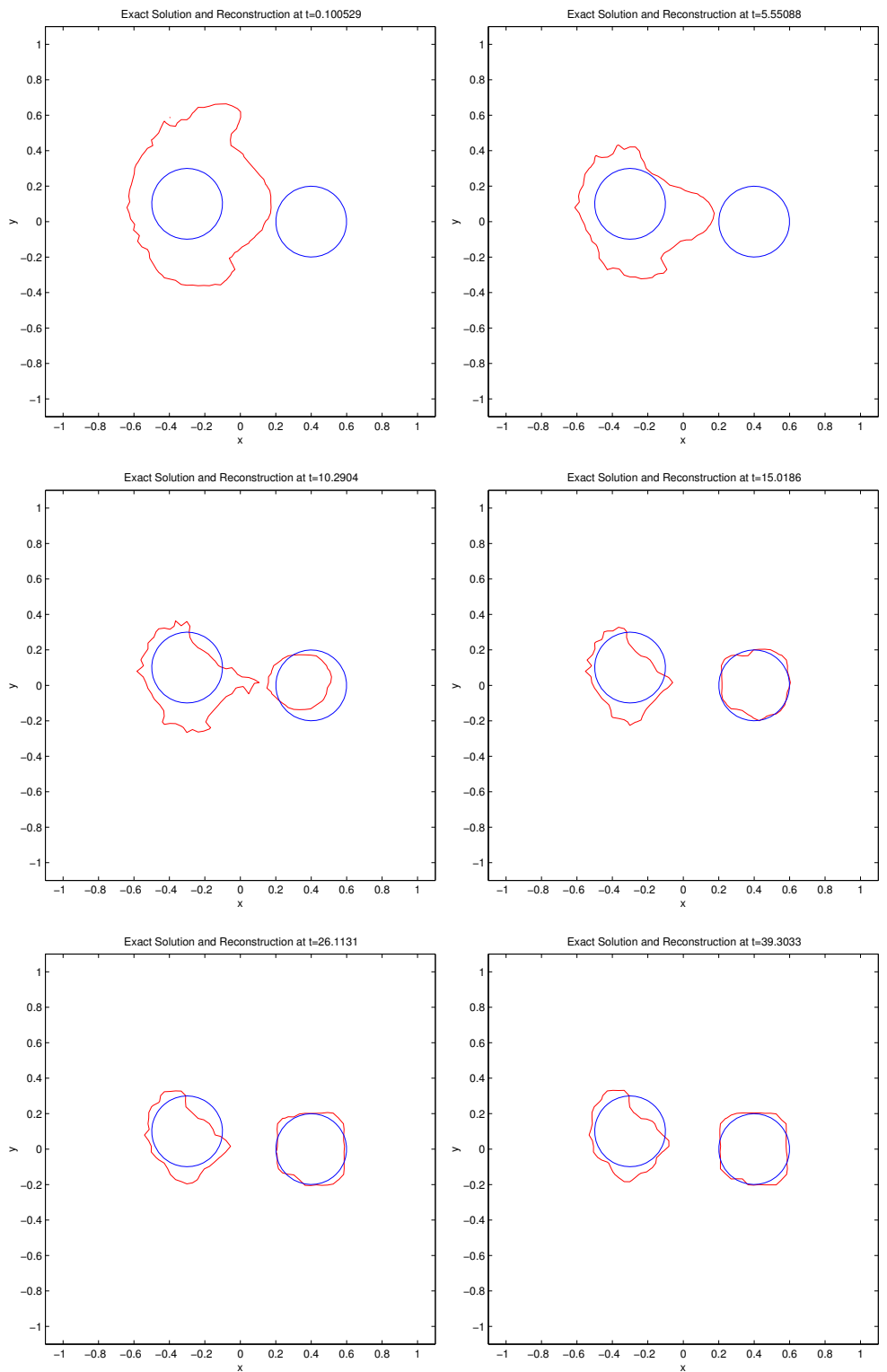

FIG. 2. Reconstructions with the Mullins-Sekerka flow at iterations 25, 50, 75, 100, 125 and 150

from iterate 20) between the exact solution and the evolving shape (i.e., the error between the corresponding indicator functions in the norm of $L^{1}(\Theta)$ ) are plotted. One observes that for stronger norms, the evolution is faster in the initial stage, before all three methods stagnate for some time. This stagnation arises before the shape splits into two connected components and is possibly due to singularities in the velocity needed for this splitting. The period over which the methods stagnate is shorter for weaker norms, so that the Mullins-Sekerka flow 

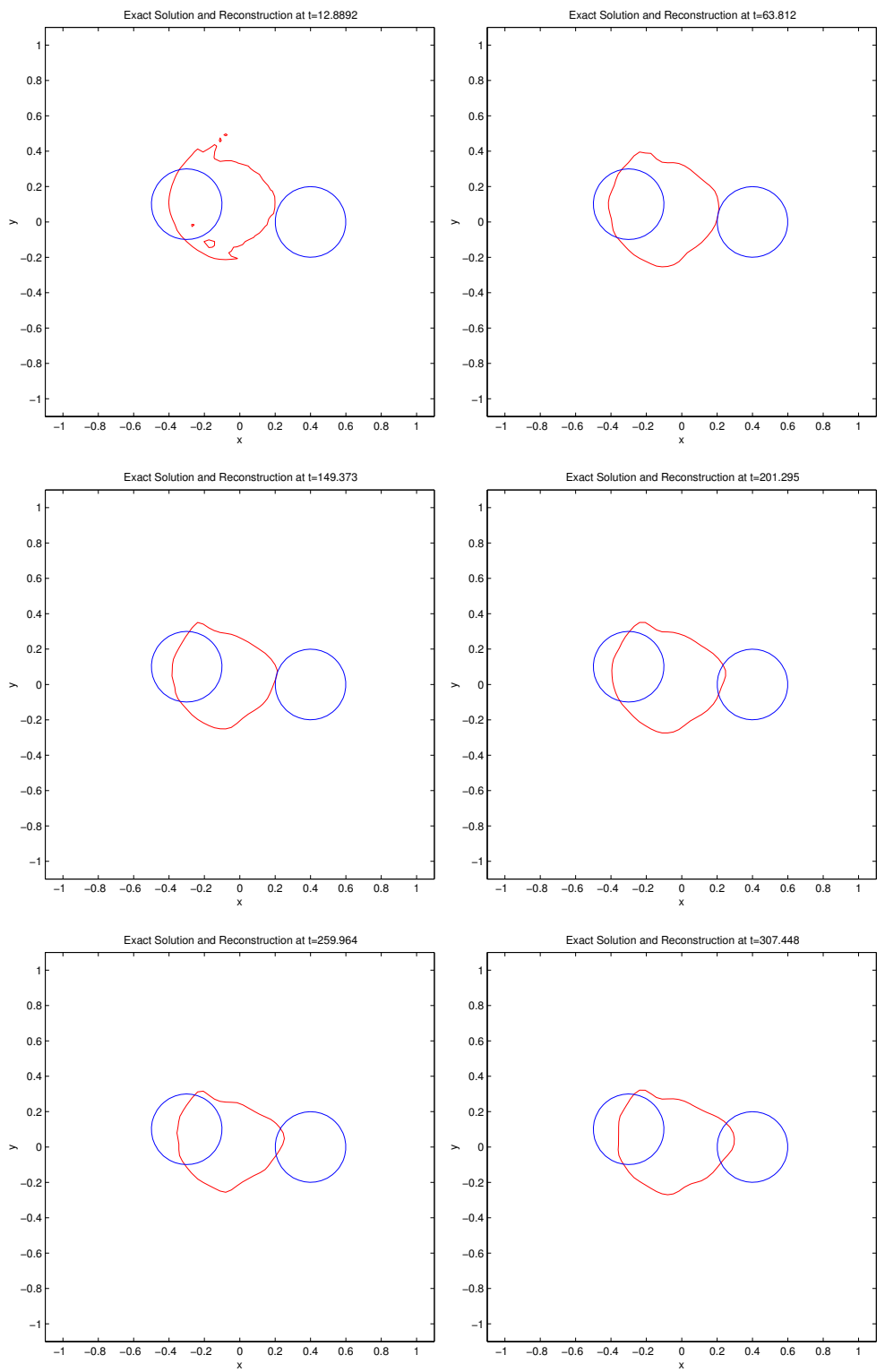

FIG. 3. Reconstructions with the Stefan-type flow at iterations 25, 50, 75, 100, 125 and 150.

finally yields the fastest evolution. As usual for iterative (or evolutive) regularization methods for ill-posed problems in presence of noise, the error decreases only to some finite value, before increasing again or starting to oscillate. This behavior is shown by all of the methods in this case, but a reasonable reconstruction can be obtained by stopping the evolution according to the generalized discrepancy principle, i.e., the first time the residual is (roughly) of the same size as the noise. 

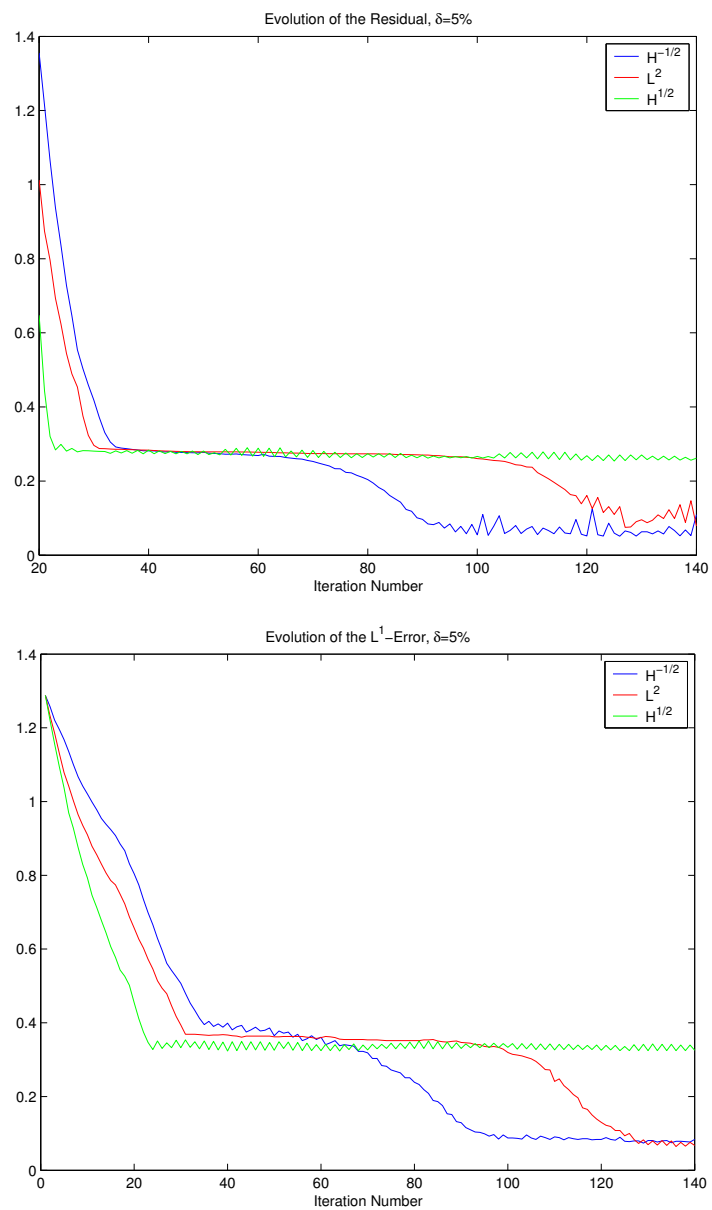

FIG. 4. Evolution of the residual (top) and error in the $L^{1}$-norm (bottom) during exact solution and reconstructed shape for $\delta=5 \%$ noise.

\subsection{Boundary and obstacle reconstruction}

For the obstacle reconstruction problem in Example 2, we have a density $\rho$ in the shape derivative consisting of a product of gradient jumps. Since we cannot expect the solution of the direct problem to be smoother than in the class $H^{1}$ (in particular for Neumann values $g$ in $H^{-1 / 2}\left(\Gamma_{n}\right)$ ), the jump of its gradient must be expected to be an element of $H^{-1 / 2}(\Omega)$. For the adjoint solution we can expect more regularity, since its Neumann values are related to the residual, which lies in $L^{2}(M)$. Nonetheless, the weakest norm in which we can expect the shape derivative to be a continuous linear form is $H^{1 / 2}(\Gamma)$ due to possible lack of regularity in the direct solution $u$.

For the numerical approximation, we use again a fictitious domain approach, which is based on the observation that the values of $u$ inside $\Omega$ have no influence on the objective in our case, and we may thus use an arbitrary continuation of $u$ inside $\Omega$. This allows us to approximate the interface 

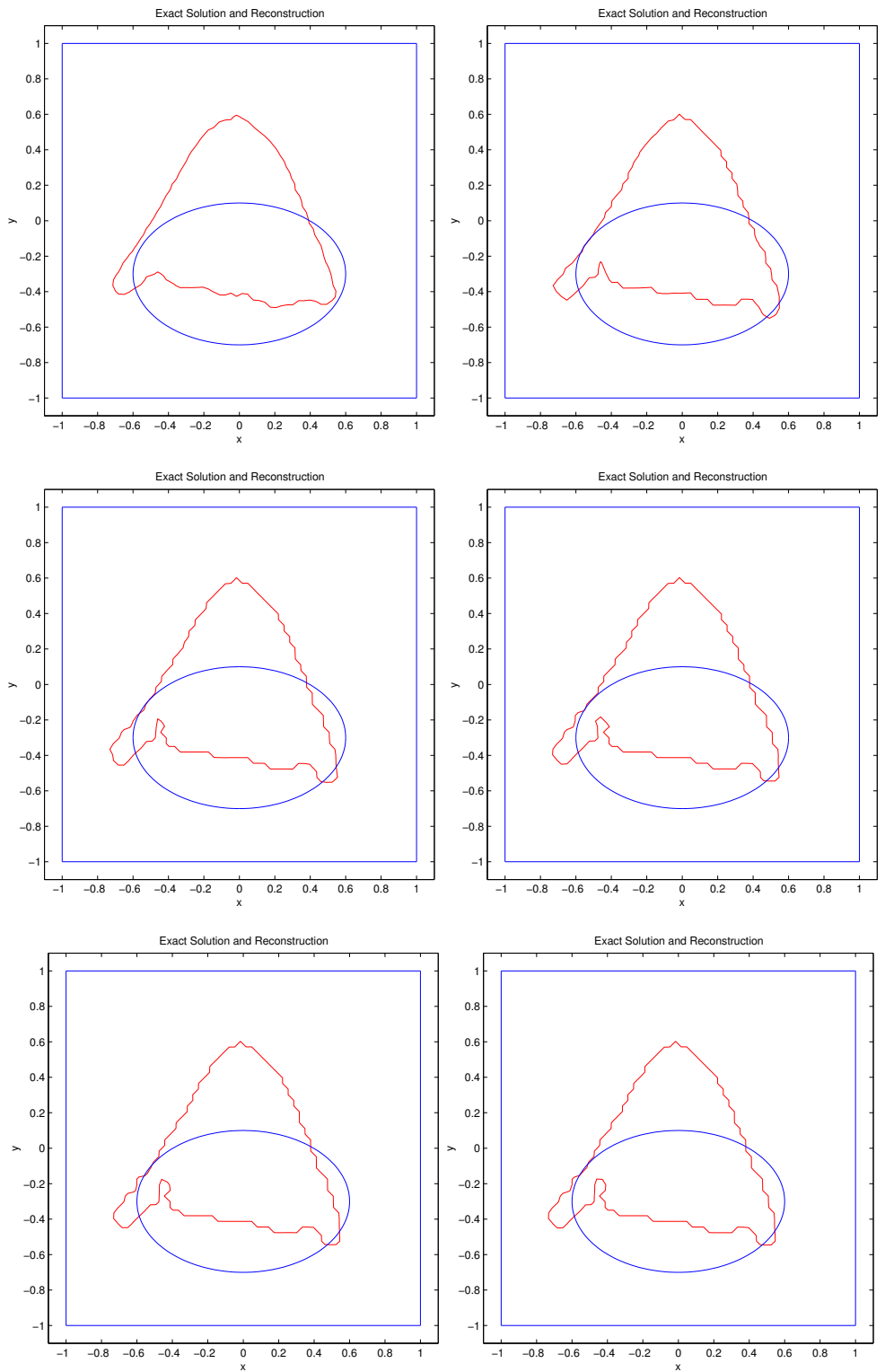

FIG. 5. Reconstructions with the Hadamard flow at iterations 50, 100, 150, 200, 250 and 300

problem by solving

$$
-\operatorname{div}\left(a_{\epsilon}(\phi) \nabla u\right)=0 \quad \text { in } \Theta
$$

subject to the original boundary conditions on $\Gamma_{n}$ and $\Gamma_{d}$, where $a_{\epsilon}$ is a function satisfying

$$
a_{\epsilon}(p)= \begin{cases}1 & \text { if } p>\epsilon \\ \epsilon & \text { if } p<-\epsilon\end{cases}
$$



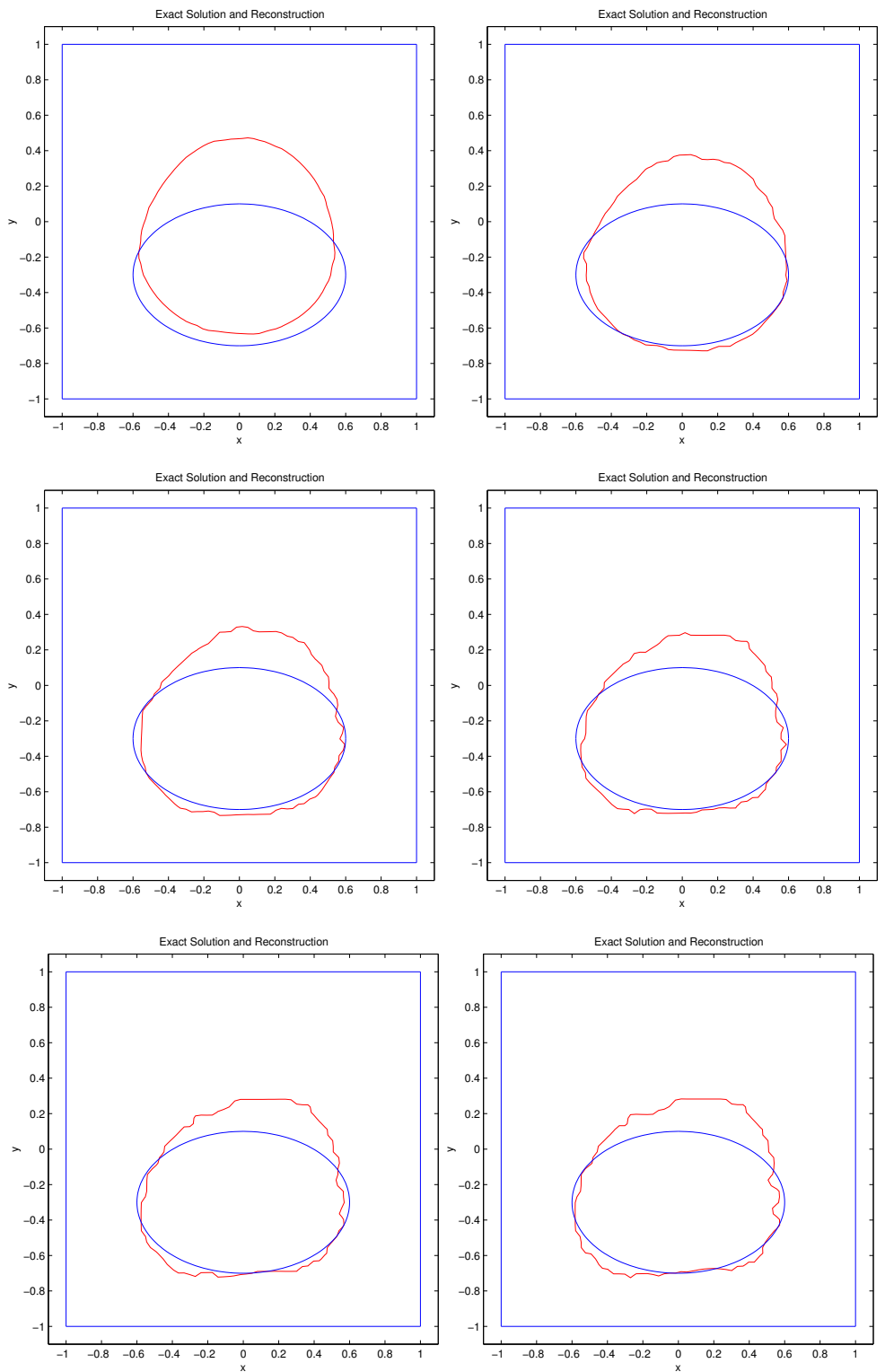

FIG. 6. Reconstructions with the Stefan-type flow at iterations 50, 100, 150, 200, 250 and 300.

and interpolates in a montone and continuous way between the values at $p=-\epsilon$ and $p=\epsilon$. This approach is frequently used in shape optimization, when Neumann boundaries are given on a shape to be optimized, and is also called weak material method. The computation of shape derivatives for the relaxed problem can be carried out as in the previous example. Moreover, we use the same discretization and solvers for the level set equation (1.7) as above. 

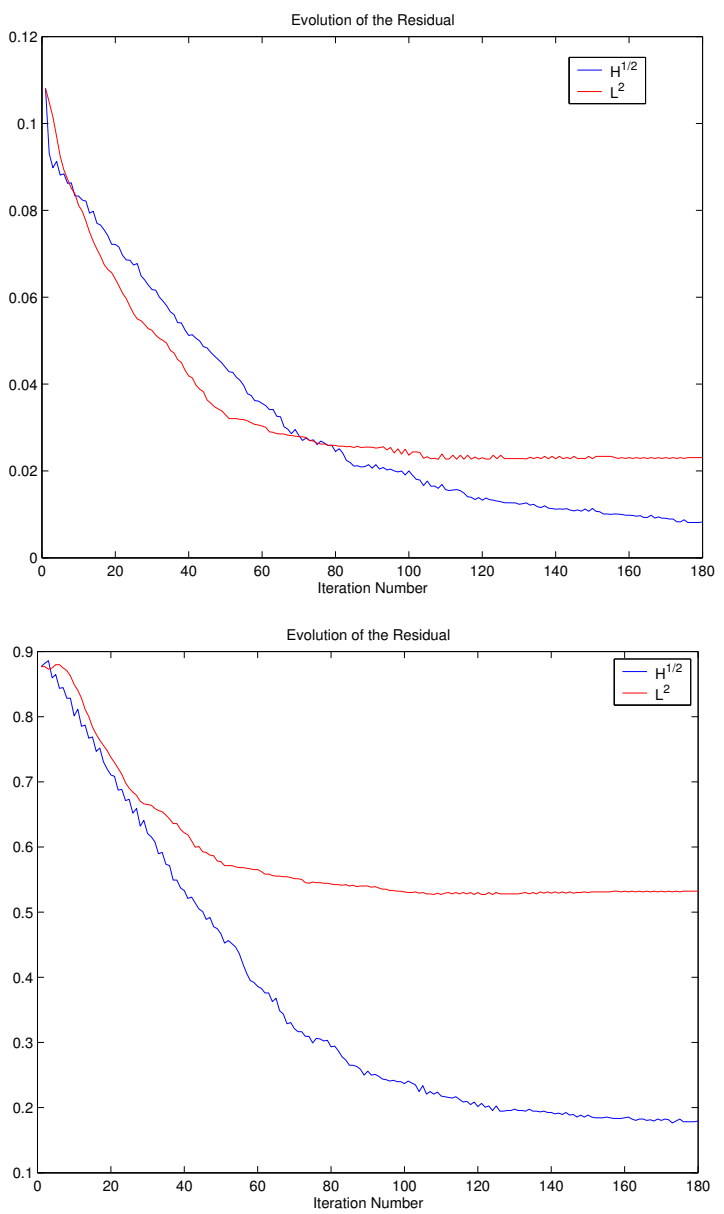

FIG. 7. Evolution of the residual (top) and error in the $L^{1}$-norm (bottom) during exact solution and reconstructed shape for $\delta=2 \%$ noise.

Our numerical test is carried out in the case of $\Theta=(-1,1)^{2}$, with homogeneous Dirichlet conditions on $\Gamma_{d}=\{1\} \times(-1,1)$ and measurements on the Neumann boundary $M=\Gamma_{n}=$ $\partial \Theta-\Gamma_{d}$. Since data are measured only on the boundary, we have to expect a severely ill-posed problem without too accurate reconstructions of the exact solution. We use Santosa's flow as well as a Stefan-type flow starting from a circle with radius 0.5 centered at $(0,0.2)$. The exact solution is an elliptic shape, plotted in Figures 5, 6, synthetic data and noise (with noise level 2\%) are generated in the same way as for the example of support reconstruction. From Figure 5 one observes that the flow constructed according to Santosa's approach shows an unstable behavior and fails to converge to the solution, while the Stefan-type flow shown in Figure 6 yields a reasonable reconstruction for this size of the noise level. This statement is confirmed by the plots of the evolving residual (top) and $L^{1}$-error (bottom) in Figure 7 , from which one observes that Santosa's flow stagnates far away from the solution, while the Stefan-type flow shows the expected convergence behavior. This result demonstrates again the importance of choosing appropriate level set evolutions, in particular 
in connection with ill-posed problems, where one needs a stable method being able to handle data noise.

\section{Conclusions and outlook}

In this paper we have provided a rather general framework for the construction of level set methods based on shape derivatives of an associated objective functional. So far, we have only considered methods in a gradient flow setup, but several extensions are possible and subject to future work, such as e.g.:

- For problems where a vanishing derivative of the objective does not imply that the level set evolution has reached an optimum, one can consider other choices of the velocity, which incorporate more information on the residual than the shape derivative. For a certain class of problems, such a choice was investigated by the author in [18].

- Instead of gradient flows one can consider continuous Newton-type methods in a level set framework. In a setup similar to the gradient flow proposed by Santosa, Newton-type approaches have been considered by several authors [22, 27, 28, 66]. An extension to other norms as proposed in this paper seems rather obvious.

- As mentioned in the introduction, further investigations on the efficient coupling of the level set evolution with the partial differential equations for the state seem to be necessary. Particularly promising seems to be a cascadic approach, since this can yield a very fast reduction of the objective during the initial stage of the evolution, when a coarse grid is used.

- A rigorous analysis of the methods proposed here, with respect to their well-posedness, their convergence and regularizing properties, is still completely open.

\section{Acknowledgments}

Useful and stimulating discussions with Prof. Heinz W. Engl, Benjamin Hackl (University Linz), Hend Benameur (ENI Tunis), and Gregoire Allaire (Ecole Polytechnique Paris) are acknowledged. Financial support of the Austrian National Science Foundation FWF under grant SFB F 013/08 is acknowledged.

\section{REFERENCES}

1. Adalsteinsson, B. \& Sethian, J. A. The fast construction of extension velocities in level set methods. J. Comput. Phys. 148 (1999), 2-22. Zbl 0919.65074 MR 99j:65189

2. Adams, R. A. Sobolev Spaces. Academic Press, New York (1975). Zbl 0314.46030 MR 56 \#9247

3. Alessandrini, G., Beretta, E., Rosset, E., \& Vessella, S. Optimal stability for inverse elliptic boundary value problems with unknown boundary. Ann. Scuola Norm. Sup. Pisa 29 (2001), 755-806. Zbl pre01884316 MR 2002b:35196

4. Alessandrini, G., Isakov, V., \& Powell, J. Local uniqueness in the inverse conductivity problem with one measurement. Trans. Amer. Math. Soc. 347 (1995), 3031-3041. Zbl 0864.35114 MR 95m:35197

5. Alessandrini, G. \& Rosset, E. The inverse conductivity problem with one measurement: Bounds on the size of the unknown object. SIAM J. Appl. Math. 58 (1998), 1060-1071. Zbl 0953.35141 MR 99j:35227 
6. Alessandrini, G., Rosset, E., \& Seo, J. K. Optimal size estimates for the inverse conductivity problem with one measurement. Proc. Amer. Math. Soc. 128 (2000), 53-64. Zbl 0944.35108 MR 2000j:35267

7. Allaire, G., Jouve, F., \& Toader, A. M. A level-set method for shape optimization. C. R. Acad. Sci. Paris Sér. I 334 (2002), 1125-1130. Zbl pre01777429 MR 2003b:49041

8. Almgren, F. \& TAYLOR, J. E. Optimal geometry in equilibrium and growth. Fractals 3 (1995), 713-723. Zbl 0885.58015 MR 97h:58054

9. Alvarez, S. A. \& LiU, C. Motion of a closed curve by minus the surface Laplacian of curvature. Differential Integral Equations 13 (2000), 1583-1594. Zbl 0974.35053 MR 2002j:35314

10. Ambrosio, L. \& Mantegazza, C. Curvature and distance function from a manifold. J. Geom. Anal. 8 (1998), 723-748. Zbl 0941.53009 MR 2001d:49070

11. BenAmeUR, H., BURGER, M., \& HACKL, B. Level set methods for some geometric inverse problems in linear elasticity. Preprint (2003).

12. Bendsoe, M. P. Optimization of Structural Topology, Shape, and Material. Springer, Berlin (1995). Zbl 0822.7300 MR 96g:73043

13. Beretta, E. \& Vessella, S. Stable determination of boundaries from Cauchy data. SIAM J. Math. Anal. 30 (1999), 220-232. Zbl 0928.35201 MR 99j:35229

14. Binder, A., HANKe, M., \& SCHERZER, O. On the Landweber iteration for nonlinear ill-posed problems. J. Inverse Ill-Posed Probl. 4 (1996), 381-389. Zbl 0879.65037| MR 97j:65096

15. BRyAn, K. \& CAUdill, L. F. An inverse problem in thermal imaging. SIAM J. Appl. Math. 56 (1996), 715-735. Zbl 0854.35125 MR 96m:80002

16. Bryan, K. \& CAUdill, L. F. Uniqueness for a boundary identification problem in thermal imaging. Electron. J. Differ. Equ. Conf. 1 (1998), 23-39. Zbl 0911.35117 MR 2000g:35226

17. Bukhgeim, A. L., Cheng, J., \& Yamamoto, M. Stability for an inverse boundary problem of determining a part of a boundary. Inverse Problems 15 (1999), 1021-1032. Zbl 0934.35202 MR 2000e:35235

18. Burger, M. A level set method for inverse problems, Inverse Problems 17 (2001), 1327-1355. Zbl 0985.35106 MR 2002g:35208

19. Burger, M., Engl, H. W., \& Markowich, P. Inverse doping problems for semiconductor devices. Recent Progress in Computational and Applied PDEs, T. F. Chan et al. (eds.), Kluwer Academic/Plenum Publishers (2002), 27-38.

20. Burger, M., Engl, H. W., Markowich, P., \& Pietra, P. Identification of doping profiles in semiconductor devices. Inverse Problems 17 (2001), 1765-1795. Zbl 0989.35139 MR 85g:35121

21. Carter, W. C., TAYlor, J. E., \& CAhn, J. W. Variational methods for microstructural evolution. J. Minerals Metals Mater. Soc. 49 (1997), 30-36.

22. Chan, T. F., Heimsund, B. O., Kastberg Nilssen, T., \& Tai, X. C. Level set methods and augmented Lagrangian for parameter identification. Preprint (2002).

23. Chopp, D. L. \& Sethian, J. A. Motion by intrinsic Laplacian of curvature. Interfaces Free Bound. 1 (1999), 107-123. Zbl 0938.65144 MR 2002j:65023

24. Colton, D. \& Kress, R. Inverse Acoustic and Electromagnetic Scattering Theory. 2nd ed., Springer, Berlin (1998). Zbl 0893.35138 MR 99c:35181

25. Delfour, M. C. \& Zolésio, J.-P. Shapes and Geometries. Analysis, Differential Calculus, and Optimization. SIAM, Philadelphia (2001). Zbl 1002.49029 MR 2002i:49002

26. Delfour, M. C. \& ZolÉSIO, J.-P. Velocity method and Lagrangian formulation for the computation of the shape Hessian. SIAM J. Control Optim. 29 (1991), 1414-1442. Zbl 0747.49007 MR 92j:49042

27. Dorn, O., Miller, E. M., \& RAPPAPORT, C. M. A shape reconstruction method for electromagnetic tomography using adjoint fields and level sets. Inverse Problems 16 (2000), 1119-1156. Z Zbl 0983.35150 MR 2002a:78025 
28. Dorn, O., Miller, E. L., \& Rappaport, C. M. Shape reconstruction in 2D from limitedview multifrequency electromagnetic data. Contemp. Math. 278 (2001), 97-122. Zbl 0988.78012 MR 2002f:35219

29. Engl, H.W., Hanke, M., \& Neubauer, A. Regularization of Inverse Problems. Kluwer, Dordrecht (1996). Zbl 0859.65054 MR 97k:65145

30. Escher, J., MAYER, U., \& SimOnetT, G. The surface diffusion flow for immersed hypersurfaces. SIAM J. Math. Anal. 29 (1998), 1419-1433. Zbl 0912.35161 MR 99f:58042

31. Federer, H. Geometric Measure Theory. Springer, Berlin (1969). Zbl 0176.00801 MR 41 \#1976

32. Friedman, A. \& IsAKov, V. On the uniqueness in the inverse conductivity problem with one measurement. Indiana Univ. Math. J. 38 (1989), 563-579. Zbl 0703.35165 MR 91a:35164

33. Groetsch, C. W. \& Hanke, M. Nonstationary iterated Tikhonov regularization. J. Optim. Theory Appl. 98 (1998), 37-53. Zbl 0910.47005 MR 99e:65100

34. Groetsch, C. W. \& Scherzer, O. Nonstationary iterated Tikhonov-Morozov method and third order differential equations for the evaluation of unbounded operators. Math. Methods Appl. Sci. 23 (2000), 1287-1300. Zbl 0970.65059 MR 2001m:65078

35. Hanke, M., Neubauer, A., \& Scherzer, O. A convergence analysis of the Landweber iteration for nonlinear ill-posed problems. Numer. Math. 72 (1995), 21-37. Zbl 0840.65049 MR 96i:65046

36. Hettlich, F. \& RUNDELL, W. Iterative methods for the reconstruction of an inverse potential problem. Inverse Problems 12 (1996), 251-266. Zb1 0621.35102 MR 97m:65232

37. Hettlich, F. \& Rundell, W. Recovery of the support of a source term in an elliptic differential equation. Inverse Problems 13 (1997), 959-976. Zbl 0888.35135 MR 98g:35213

38. Hettlich, F. \& Rundell, W. The determination of a discontinuity in a conductivity from a single boundary measurement. Inverse Problems 14 (1998), 67-82. Zbl 0894.35126 MR 99b:35210

39. HoffmAnn, K. H. \& SpRekels, J. On the identification of coefficients of elliptic problems by asymptotic regularization. Numer. Funct. Anal. Optim. 7 (1984), 157-177. Zbl 0576.65121 MR 86c:35161

40. Hoffmann, K. H. \& Sprekels, J. On the identification of parameters in general variational inequalities by asymptotic regularization. SIAM J. Math. Anal. 17 (1986), 1198-1217. Zbl 0652.35103 MR 87j:35373

41. Howison, S. D. \& Ockendon, J. R. (eds.) Special issue: Hele-Shaw flows and related problems. Eur. J. Appl. Math. 10 (1999), 511-705. Zbl 0951.00024 MR 2001a:76005

42. ISAKOV, V. \& POWELL, J. On the inverse conductivity problem with one measurement, Inverse Problems 6 (1990), 311-318. Zbl 0724.35108 MR 91e:35212a

43. Ito, K., KUniSch, K., \& LI, Z. Level-set function approach to an inverse interface problem. Inverse Problems 17 (2001), 1225-1242. Zbl 0986.35130 MR 2002h:35335

44. JiAnG, G. S. \& PENG, D. Weighted ENO-schemes for Hamilton-Jacobi equations. SIAM J. Sci. Comput. 21 (2000), 2126-2143. Zbl 0957.35014 MR 2001e:65124

45. Kang, H., Seo, J. K., Jin, K., \& SheEn, D. Numerical identification of discontinuous conductivity coefficients. Inverse Problems 13 (1997), 113-123. Zbl 0934.35204 MR 98k:78052

46. Kaup, P., Santosa, F., \& Vogelius, M. A method for imaging corrosion damage in thin plates from electrostatic data. Inverse Problems 12 (1996), 279-293. Zbl 0851.35134

47. KUnisCh, K. \& PAN, X. Estimation of interfaces from boundary measurements. SIAM J. Control Optim. 32 (1994), 1643-1674. Zbl 0807.35162 MR 95h:35245

48. Landweber, L. An iteration formula for Fredholm integral equations of the first kind. Amer. J. Math. 73 (1951), 615-624. Zbl 0043.10602 MR 13,247c

49. Lions, J. L. \& Magenes, E. Non-Homogeneous Boundary Value Problems and Applications. Springer, Berlin (1972). Zbl 0227.35001 MR 50 \#2670 
50. Litman, A., Lesselier, D., \& Santosa, F. Reconstruction of a two-dimensional binary obstacle by controlled evolution of a level-set. Inverse Problems 14 (1998), 685-706. Zbl 0912.35158 MR 99e:78032

51. Milic, N. On the Mullins-Sekerka model for phase transitions in mixtures, Quart. Appl. Math. 49 (1991), 437-445. Zbl 0742.35075 MR 92i:80005

52. Mohammadi, B. \& Pironneau, O. Applied Shape Optimization for Fluids. Clarendon Press, Oxford (2001). Zbl 0970.76003 MR 2002d:76001

53. Morel, J. M. \& Solimini, S. Variational Methods in Image Segmentation. With Seven Image Processing Experiments. Birkhäuser, Basel (1994). Zbl 0827.68111 MR 96b:68184

54. Mullins, W. W. \& SeKerka, R. F. Morphological stability of a particle growing by diffusion or heat flow. J. Appl. Phys. 34 (1963), 323-329.

55. Novruzi, A. \& Pierre, M. Structure of shape derivatives. J. Evol. Equ. 2 (2002), 365-382. MR 1930612

56. Osher, S. \& FedKIw, R. P. The Level Set Method and Dynamic Implicit Surfaces. Springer, New York (2003). Zbl pre01873119

57. Osher, S. \& SAntosA, F. Level set methods for optimization problems involving geometry and constraints I. Frequencies of a two-density inhomogeneous drum. J. Comput. Phys. 171 (2001), 272-288. Zbl pre01655220 MR 2002f:65088

58. Osher, S. \& A. Sethian, J. Fronts propagating with curvature-dependent speed: algorithms based on Hamilton-Jacobi formulations. J. Comput. Phys. 79 (1988), 12-49. Zbl 0659.65132 MR 89h:80012

59. M. PARK, H. \& J. Shin, H. Shape identification for natural convection problems using the adjoint variable method. Preprint (2002).

60. Pironneau, O. Optimal Shape Design for Elliptic Systems. Springer, New York (1984). Zbl 0534.49001 MR 86e:49003

61. Radmoser, E., Scherzer, O., \& Weickert, J. Scale-space properties of nonstationary iterative regularization methods. J. Vis. Commun. Image Repr. 8 (2000), 96-114.

62. RADMOSER, E. \& WINCOR, R. Determining the inner contour of a furnace from temperature measurements. Technical Report 12/1998, Industrial Mathematics Institute, University of Linz (1998).

63. Ramananjaona, C., Lambert, M., \& Lesselier, D. Shape inversion from TM and TE real data by controlled evolution of level sets. Inverse Problems 17 (2001), 1585-1595. Zbl 0987.78500 MR 1872909

64. Ramananjaona, C., LAmbert, M., Lesselier, D., \& Zolésio, J.-P. Shape reconstruction of buried obstacles by controlled evolution of a level set: from a min-max formulation to numerical experimentation. Inverse Problems 17 (2001), 1087-1111. Zbl 0994.35121 MR 1861503

65. RUnSt, T. \& SiCKEL, W. Sobolev Spaces of Fractional Order, Nemytskij Operators and Nonlinear Partial Differential Equations. de Gruyter, Berlin (1996). Z Zbl 0873.35001 MR 98a:47071

66. Santosa, F. A level-set approach for inverse problems involving obstacles. ESAIM Contrôle Optim. Calc. Var. 1 (1996), 17-33. Zbl 0870.49016 MR 97f:00011

67. SCHERZER, O. Convergence criteria of iterative methods based on Landweber iteration for solving nonlinear problems. J. Math. Anal. Appl. 194 (1995), 911-933. Zbl 0842.65036 MR 97d:65033

68. Scherzer, O. \& Weickert, J. Relations between regularization and diffusion filtering. J. Math. Imaging Vision 12 (2000), 43-63. Zbl 0945.68183 MR 2000k:68167

69. Sethinan, J. A. Level Set Methods and Fast Marching Methods. 2nd ed., Cambridge Univ. Press, Cambridge (1999). Zbl 0973.76003 MR2000c:65015

70. Sethian, J. A. \& Wiegmann, A. Structural boundary design via level set and immersed interface methods. J. Comput. Phys. 163 (2000), 489-528. Zbl 0994.74082 MR 2001h:74074

71. SmereKA, P. Semi-implicit level set methods for motion by mean curvature and surface diffusion. Preprint. 
72. SokoŁowski, J. \& ZolÉsio, J.-P. Introduction to Shape Optimization. Springer, Berlin (1992). Zbl 0761.73003 MR 94d:49002

73. Sorli, K. \& SkAar, I. M. Monitoring the wear-line of a melting furnace. Proc. '3icipe' Third Internat. Conference on Inverse Problems in Engineering (Port Ludlow, 1999). http://www.me.ua.edu/3icipe/Papers.htm.

74. TAUtenhahn, U. On the asymptotical regularization of nonlinear ill-posed problems. Inverse Problems 10 (1994), 1405-1418. Zbl 0828.65055 MR 95j:65069

75. TAYlor, J. E. Surface motion due to crystalline surface energy gradient flows. Elliptic and Parabolic Methods in Geometry, A. K. Peters, Wellesley (1996), 145-162. Zbl 0915.49024 MR 97h:49054

76. Tornberg, A. K. \& EngQuist, B. Regularization techniques for numerical approximation of PDEs with singularities. CAM Report 02, UCLA (2002).

77. ZolÉSIO, J.-P. The material derivative (or speed) method for shape optimization. Optimization of Distributed Parameter Structures, Vol. II, NATO Adv. Study Inst. Ser. E, Appl. Sci. 50, Nijhoff, The Hague (1981), 1089-1151. Zbl 0517.73097 MR 84f:49017 